\title{
Proportional Study of TCP Variants over Heterogeneous Wireless Networks
}

\author{
Abdul Razaque \\ Computer Science Department \\ University of Bridgeport \\ CT-06604, USA
}

\author{
Khaled Elleithy \\ Computer Science Department \\ University of Bridgeport \\ CT-06604, USA
}

\begin{abstract}
Transmission control protocol (TCP) was initially designed for static networks to provide the consistent data delivery. The enhancement of TCP performance was also achieved with different types of networks with the introduction of new TCP deviations. However, there are still many factors that affect the performance of TCP. Mobility is one of the primary performance-affecting drivers in heterogeneous network. The research of state in this paper is to determine the best TCP variant from the mobility point of view. We simulate some TCP variants such as TCP-Tahoe, TCP-Reno, TCP-New Reno, TCP- Vegas, TCP-SACK and TCP-Westwood from the mobility point of view.

The scenarios presented in this paper are supported by ondemand link weight (ODLW) routing protocol that helps find the efficient route with Quality of Service (QoS) provisioning. The scenarios are designed for the walking persons to vehicles, and particularly suited for rocky and deserted areas. To demonstrate the strength of these TCP variants, the scenarios are simulated and evaluated the QoS parameters such as round trip time (RTT) fairness, end-to-end delay, and the number of broken links. Finally, based on the outcomes, we identify the best TCP variant that could be used for several applications in the future over heterogeneous wireless networks.
\end{abstract}

\section{General Terms}

Wireless communication networks, experiments and simulation.

\section{Keywords}

TCP variants, heterogeneous network, RTT, throughput, broken links, end-to-end delay

\section{INTRODUCTION}

The fact is that the TCP was originally designed for the wired network, and even its good performance is in the wired network[1].The researchers have put their efforts for improving the performance of TCP in wireless networks, and all this work confirmed that TCP in its present structure is not appropriate for MANET[2]. TCP has high overhead due to packet loss that may cause the buffer congestion because the MANET's losses is due to an error or the frequently of the mobility [3]. So, the TCP cannot be efficient in current form[4]. Furthermore, the main reason of the weak performance of the standard TCP in the wireless network is the inability of discovering the lost packets[5]. So, we can realize that both characteristics have the same reason of packet loss, which is the based on the network congestion[6]. However, some new protocols have been introduced and implemented to avoid packet loss due to congestion. We evaluate some of the existing TCP variants, and then demonstrate how they in a heterogeneous network particularly MANET.

The MANET network is a particular network due to the ability of its nodes to communicate with each other via intermediate nodes. So, it can be set up in any remote areas without infrastructure-based support. The nodes, which are the part of MANET require the data and information from the database. However, the database is available in a wired network. Therefore, MANET can be integrated with wired network to obtain the required data and information [7].

Some applications run over the database, which are supported by TCP variants. This paper aims to exhibit the weaknesses and strengths of TCP variants in the heterogeneous network especially MANET. Simulation is conducted using NS2. TCP variants use different congestion control mechanisms, which include congestion window, recovery mechanism, retry limit, maximum packet size and backup mechanism[8]. To determine the performance for each TCP variants, as new architecture and approaches are required to find out complete behavior of the variants. This motivation results to introduce such network to analyze the effectiveness for each TCP Variants. The random waypoint mobility model is also used for controlling the moments of nodes. In order to analyze the impact of mobility in the heterogeneous network, it is essential that underlying mobility model should attain the realistic scenario or at least significant features. To this conclusion, we deem that this paper makes the reasonable contribution. The scope of this paper is to analyze some existing TCP variants over heterogeneous network. The focus of study is particularly around the performance metrics such as throughput, RTT fairness, end-to-end delay, and broken links in presence of the mobility.

The remaining paper is organized as follows. In Section 2, we present related work. In Section 3, we present heterogeneous network design. In Section 4, we explain handover process. In Section 5, we present simulation setup and analysis of the result. In Section 6, we present the discussion of the results and finally section 7 concludes the work and future directions.

\section{RELATED WORK}

In this section, we present the salient features of existing TCP variants. The performance of TCP Reno, New Reno, SACK and Tahoe was evaluated in [9]with respect to goodput under three routing protocols over static multi-hop network. The authors demonstrated that Reno had edge over other TCP variants. However, the scenario was not fully explained. Our work is entirely different from their work because we use 
ODLW protocol in heterogeneous network with the inclusion of additional TCP variants; Vegas and Westwood. Furthermore, our work discusses relatively all QoS parameters. In our previous work [3], the performance of TCP variants was compared deploying anchor point node (APN) heterogeneous network using dynamic source routing protocol. However, the previous work focused on the throughput, and packet delivery ratio. The finding of this paper gives complete knowledge about the behavior of each TCP Variants in a heterogeneous environment. We narrowly analyze all issues of MANET in presence of mobility and showed their impact on the performance of each TCP Variants. The static scenario of heterogeneous network is simulated using the TCP Reno, TCP SACK and TCP Westwood in [8]. The authors claimed that TCP Westwood was better performer, whereas our work completely depends on movement and speed of the nodes with Random waypoint model. Moreover, we have thoroughly studied the behavior of TCP Variants.

\section{HETEROGENEOUS NETWORK DESIGN}

We have designed mobility-based scenarios in heterogeneous network by combining the features of wired with wireless and MANET networks to make reasonable communication even in remote areas. The nodes, which make the possible communication between different segments of the network are called gateway (APN). The APN can play a role as coordinator in the network. Three segments of networks are jointly connected to make the heterogeneous network. The APNs are located on different positions.

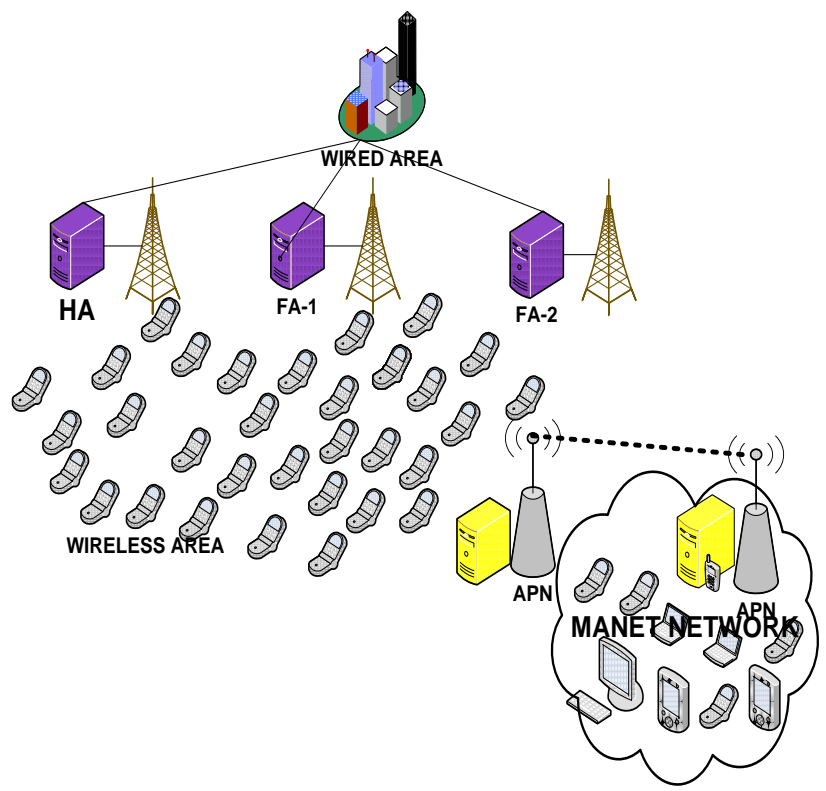

Fig 1: Heterogeneous Network Design

The APN of MANET has information about the nodes, and these nodes are assigned the IPs locally through Dynamic Host Configuration Protocol Server (DHCP). The APN that is part of MANET is said as MANET anchor point node (MAPN). Similarly, the node that is located at the area where wireless range becomes weak is called Infrastructure-based anchor point node (IBAPN). Both APNs can play a role as coordinators and formulate possible communication for the rest of nodes in fixed and MANET segment of the network.

The wired and wireless segments of network cover the urban and suburbs areas of the urban environment and MANET portion of the network covers the remote areas. The MANET network is routed using ODLW protocol. We have created twenty five traffic flows simultaneously to analyze the performance of TCP variants. This heterogeneous network could be suited for providing the healthy communication for urban as well as remote areas depicted in Figure1.

\section{HANDOVER PROCESS}

This section presents an outline of initial connection setup and handover process for MANET mobile node (MMN). Figure 2 shows the timing diagram and describes the signals involved in it. Initial connection setup and MMN handover process can be defined by using following steps. Initially the nodes, which are the part of MANET, establish the communication with the corresponding node $(\mathrm{CN})$. The nodes establish the initial connection-setup and also send a message through Current MANET anchor point node (CMAPN). The process of connection-setup is described in following steps:

a. Once CMAPN obtains the request for connectionsetup from MMN, then it forwards the message "coordination request for connection setup" to infrastructure-based anchor point node (IBAPN). In response CMAPN also sends back message "Reply for connection setup with CN" to MMN. When MMN obtains the message from CMAPN, then it waits until the initial connection is established.

b. IBAPN forwards the message "forwarding coordination request for connection setup" to respective HA/FA within wired area. HA, /FA informs the IBAPN with message "accept coordination request" to CMAPN.

c. HA, /FA forwards message with "forwarding initial connection setup" to $\mathrm{CN}$ and sends back response to IBAPN "accept forwarding coordination request". When $\mathrm{CN}$ receives the message, then it informs the HA/FA "accept initial connection setup" with (MMN). With the establishment of initial connection setup between $\mathrm{CN}$ and MMN then, data exchange process is started.

d. When MMN changes the location and moves to another MANET then, it sends a request for handover to new MANET anchor point node (NMAPN) with the message "request for joining".

e. NMAPN sends the message "location change forwarding message" (LCFM) to IBAPN for informing the handover process and similar message is forwarded to HA/FA, and finally to $\mathrm{CN}$ for location update.

f. NMAPN forwards the LCFM to IBAPN and also "update" to CMAPN. In response, CMAPN sends acknowledgment (ACK) to NMAPN for the location update.

g. When CN gets the message LCFM then, it sets the connection again with MMN and message is forwarded with "new connection setup in change of location".

h. With the establishment of the new connection, the data exchange process is initiated. 


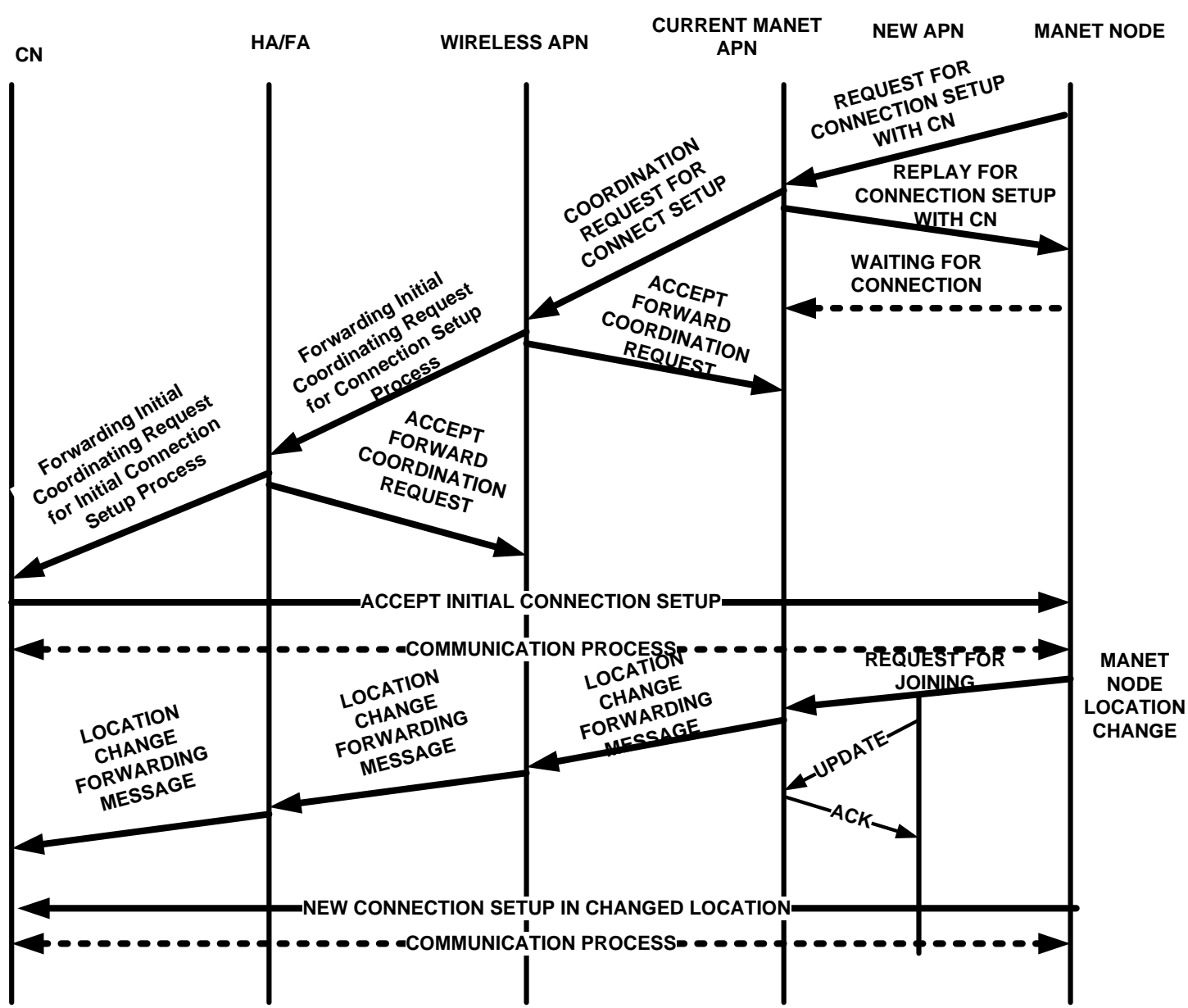

Fig 2: Handover timing process

\section{SIMULATION SETUP AND}

\section{ANALYSIS OF RESULT}

The purpose of this research is to investigate the results of TCP variants under mobility-based heterogeneous network. In this paper, we have critically evaluated the Performance of TCP variants with respect to different mobility ratios and analyzed the behavior of each TCP variant. The parameters of interest include throughput, RTT fairness, in-order data delivery, and control overhead. NS2.35 on redhat-10 is used for simulation. The random waypoint mobile scenario is generated. The simulator gives a proper model for signal propagation and transmission range is 250 meter [11]. The sensing and interference range is 550 meters. TCP New Reno, Reno, Tahoe, SACK, Westwood and Vegas are simulated and investigated on the same network so as to ensure fairness and behavior of the TCP variants. The moving speed of a node is randomly obtained through uniform division [Vmin, Vmax].

The percentage of mobility means how many mobile nodes move and resulting how many links break in the MANET. ODLW routing protocol is used to route the data, which gives better performance for routing in MANET and produces minimum routing overhead. The rest of simulated parameters are shown in Table 1.
Table 1: Simulated parameters in scenario

\begin{tabular}{|c|c|}
\hline Name of parameters & Description \\
\hline Transmission Range & 250 meters \\
\hline $\begin{array}{c}\text { Sensing and interference } \\
\text { range }\end{array}$ & 550 meters \\
\hline Bandwidth of node & $400 \mathrm{~Kb} / \mathrm{Sec}$ \\
\hline Number of nodes & 225 \\
\hline Size of network & $2400 * 1800$ square meters \\
\hline Size of MANET & $400 * 400$ square meters \\
\hline Buffering capacity & $\begin{array}{l}100 \text { Packets buffering capacity } \\
\text { at each node }\end{array}$ \\
\hline Packet generating rate & 4 packets/second \\
\hline Data Packet size & 1024 bytes \\
\hline Simulation time & 18 minutes \\
\hline Initial pause time & 30 Seconds \\
\hline MAC protocol & IEEE802.11 \\
\hline Protocols & $\begin{array}{c}\text { TCP Vegas, TCP SACK, TCP } \\
\text { NEW RENO, TCP RENO and } \\
\text { TCP TAHOE }\end{array}$ \\
\hline Mobility model & $\begin{array}{c}\text { Random waypoint mobility } \\
\text { model }\end{array}$ \\
\hline $\begin{array}{c}\text { Mobility (Speed of the } \\
\text { nodes) }\end{array}$ & $10 \mathrm{~m} / \mathrm{sec}, 20 \mathrm{~m} / \mathrm{sec}$ \\
\hline Routing Protocol & ODLW \\
\hline Mobility & $50 \%$ \\
\hline
\end{tabular}




\subsection{Throughput at different mobility rates}

We have simulated the scenarios over heterogeneous network and analyzed the performance of TCP variants (Reno, Vegas, New Reno, Tahoe, Westwood and SACK). We have counted the acknowledged packets for each TCP variant and analyzed the throughput performance. The Figure 3 shows the throughput performance for each TCP Variant at the maximum speed of $10 \mathrm{~m} / \mathrm{sec}$ with random waypoint mobility model. The performance gradually decreases of each TCP variant from $5 \%$ to $50 \%$ moving nodes. The reasons for decreasing the rate of delivered packet is the mobility. Since two nodes move in the network that fewer links break and take less time to recover, whereas $50 \%$ moving nodes cause more time to recover from broken links. The mobility also degrades the performance of TCP variants because mobility causes the change of routing information in the network.

As a result, it causes long RTT and repeated timeouts. Due to mobility, the receiver produces out-of-order segments and in resulting, the receiver generates acknowledgments (Ack) only for highest in-order-packets. This causes the duplicate Acks. The reason of delivering the more packets for TCP Vegas is that TCP Vegas retransmits the lost packets after receiving the two duplicate acknowledgments. Whereas competing TCP variants retransmit the segments after receiving the three duplicate acknowledgments but in some cases third (dupack) takes either long time or does not receive third dupack and timeout expires. It is an advantage of Vegas over competing TCP variants. Furthermore, TCP Vegas mostly retransmits the lost segments before retransmission timeout (RTO). The other reason is that TCP Vegas does not wait for the loss to trigger congestion window (cwnd) reduction.

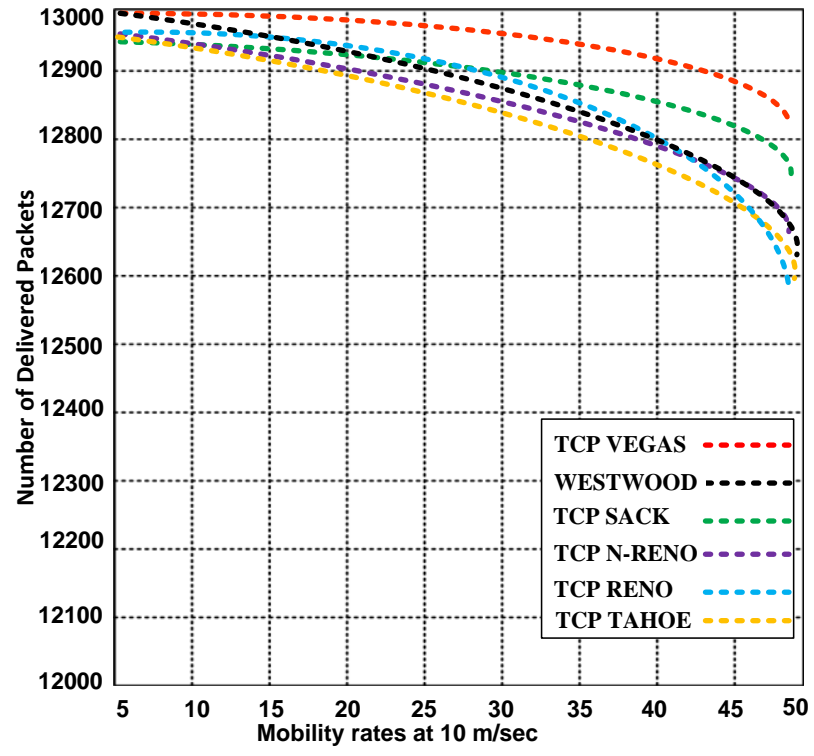

Fig 3: Number of delivered packets at different mobility rates with $10 \mathrm{~m} / \mathrm{sec}$ speed

Vegas possesses interesting approach regarding the congestion because it estimates the level of congestion before it occurs rather than attempting to avoid it. The level of congestion is measured based onm the sample RTT and size of sending window that is also the reason; the sender estimates the current throughput against every RTT. TCP Tahoe and Reno have delivered less packets than rest of TCP variants. TCP Tahoe faces the problem due to repeatation of the slow-start phases on each dropped segment, particularly when error is transient and not constant. In this case congestion window shrinks and bandwidth cannot fully be utilized. Fast Recovery algorithm for TCP New Reno can degrade the performance due to multiple losses of packets during the single window because fast recovery algorithm can manage only single loss per RTT[12].

TCP Tahoe and Reno variants have more difficulty to differentiate between loss and congestion in the wireless environment in IEEE 802.11.The performance degrading factor for TCP Reno and Tahoe is also the size of the congestion window because these variants cannot send data during the timeout period, if mainly packets loss occurs[13].

TCP Reno and Tahoe avoid timeouts in case of multiple consecutive losses occur. The major factor of degrading the performance for TCP Tahoe has no support of fast recovery algorithm. Figure 4 shows the throughput performance of TCP Variants at the maximum speed of $20 \mathrm{~m} / \mathrm{sec}$. The performance is affected by increasing the speed, and TCP New Reno is severely affected.The reason of the weak performance for TCP New Reno is also aggressive behavior of fast retransmission algorithm whenever duplicate acknowledgment (dupack) are received, and high mobility of nodes is available. Due to aggressive behavior of fast retransmission algorithm, it is difficult to deliver the packets even partial Acks are received to sender. Multiple losses due to high mobility make the weak performance of TCP New Reno because multiple losses cannot be handled properly. As a result, the network becomes more congested, and packets start to drop quickly and this claim is already verified in [14]. Another performance degrading factor relates to TCP New Reno is to take one RTT to perceive each packet loss.

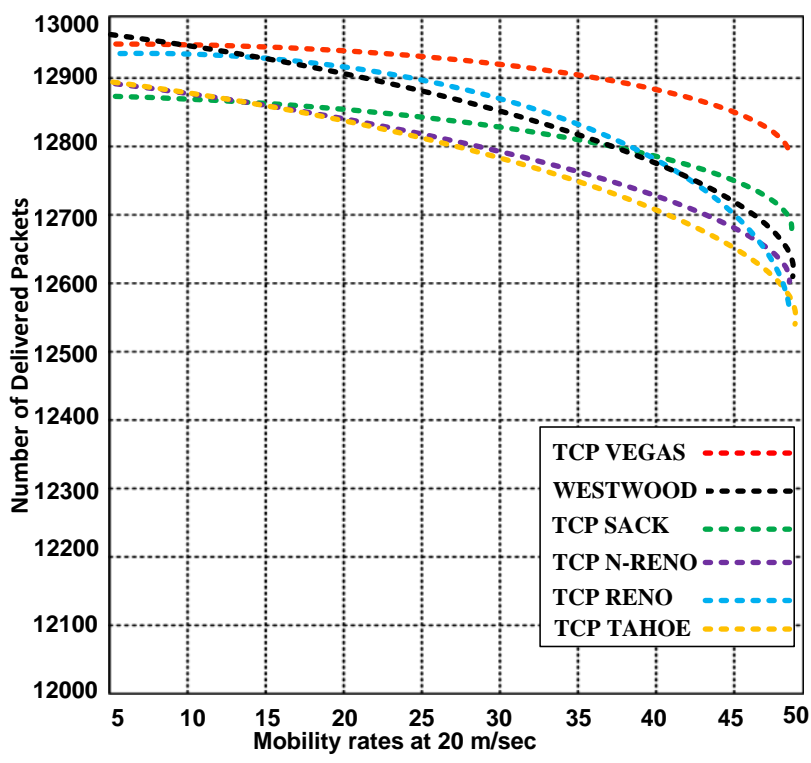

Fig 4: Number of delivered packets at different mobility rates with $20 \mathrm{~m} / \mathrm{sec}$ speed

\subsection{Round Trip Time (RTT) Fairness of TCP Variants}

We show fairness by sharing the bandwidth among different TCP variants based on the round trip time (RTT) depicted in Figure 5. There are numerous reasons for RTT fairness as one reason is to attain the equal bandwidth allocation where the different competing flows may allocate similar bottleneck. Long RTT consumes more resources than short RTT in consequence long RTT produces discouraging 
throughput. TCP Vegas gets higher throughput than other TCP variants because slow start and congestion recovery algorithms mostly influence the throughput[15].

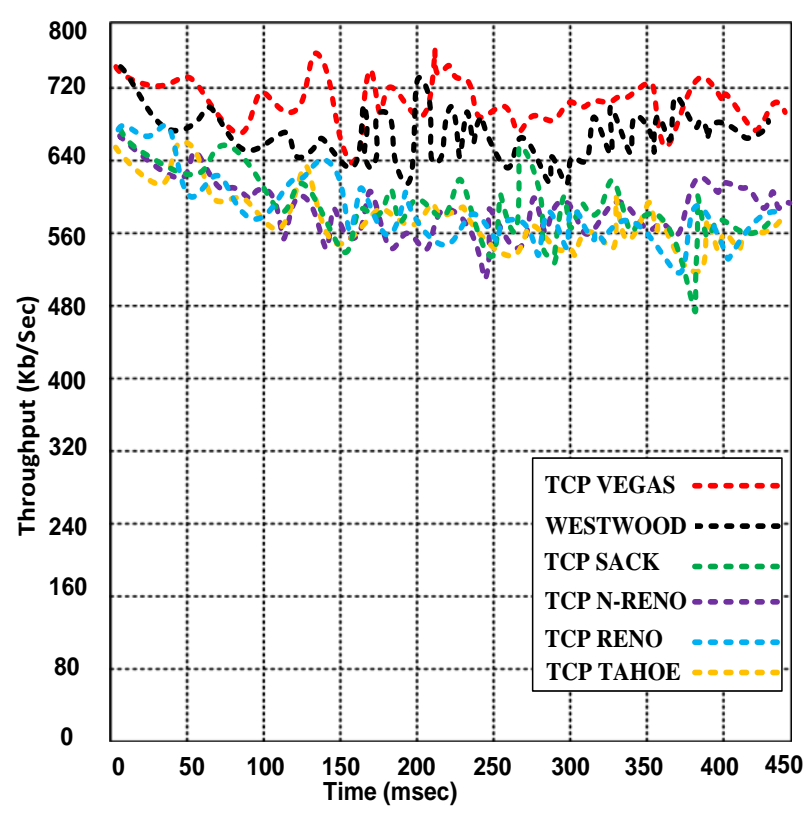

Fig 5: RTT fairness of TCP variants

Hence, slow start and congestion recovery mechanism work in a different way for each TCP variant because TCP Vegas depends on the difference of expected and actual throughput. The multiple losses can be retained by avoiding timeouts because the TCP Vegas retransmits the lost segments after receiving 2 (dupack). As, this is the reason before timeouts, dropped segments are retransmitted, and better throughput is obtained. Original feature for TCP Vegas is its congestion detection mechanism because it shows the problems concerning to fairness.

In congestion avoidance, the congestion detection algorithm of TCP Vegas verifies every RTT that is a benefit of TCP Vegas over the rest of TCP variants. Moreover, TCP Tahoe, TCP Reno, TCP New -Reno and SACK reduce the congestion windows more than once during the single RTT. This is also the reason for unfairness and producing minimum throughput, whereas RTT of Vegas reduces only once during the RTT.

\subsection{ENE TO-END DELAY at $10 \mathrm{~m} / \mathrm{sec}$ and $20 \mathrm{~m} / \mathrm{sec}$}

End-to-End delay is the average elapsed time for delivery of individual data packets. All possible delays are included and caused by routing discovery, transmission at the MAC layer and queuing at the interface queue, etc. But successfully delivered packets are calculated. We show trend for each TCP variant in Figures 6 and 7. Vegas has minimum end-to-end delay at the speed of $10 \mathrm{~m} / \mathrm{sec}$ and $20 \mathrm{~m} / \mathrm{sec}$; whereas TCP Reno and TCP Tahoe have almost similar maximum end-toend delay at $10 \mathrm{~m} / \mathrm{sec}$. As, at the speed of $20 \mathrm{~m} / \mathrm{sec}$, the maximum delay has been noticed for TCP New Reno.

The reason for maximum end-to-end delay for TCP Reno and TCP Tahoe at the speed of $10 \mathrm{~m} / \mathrm{sec}$ is weakness of faster transmission algorithms. Since, TCP Tahoe does not send instant ACKs and depends on commutative ACKs. Therefore, when packet is lost then it waits for timeout or pipeline is emptied. It causes the high bandwidth -delay.

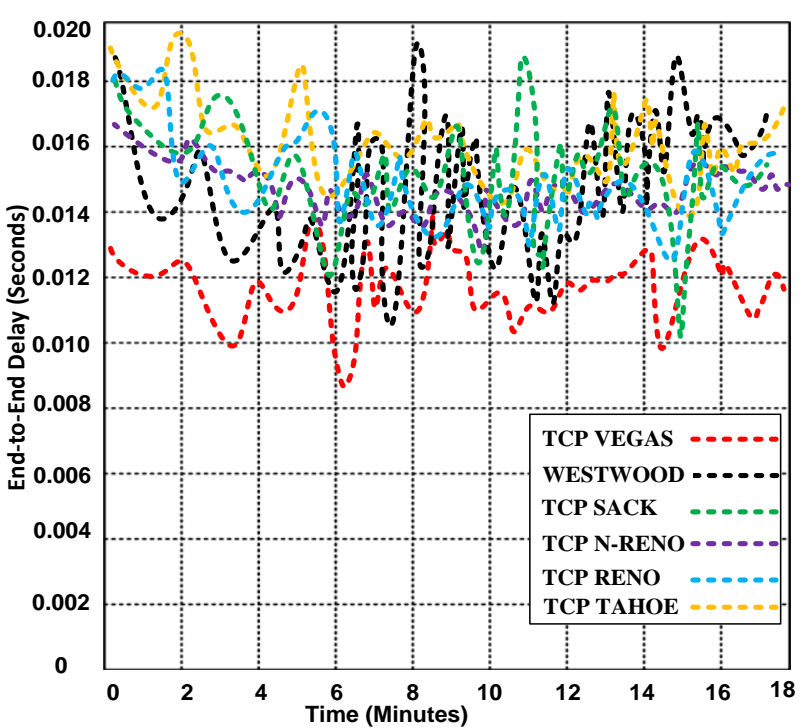

Fig 6: Average End-to-End delay of each TCP Variants at the maximum speed of $10 \mathrm{~m} / \mathrm{sec}$

TCP Reno behaves like TCP Tahoe whenever multiple losses occur and multiple losses are perceived as single segment losses. Another problem occurs with TCP Reno when the size of the window is small; number of duplicates ACKs are not detected for fast retransmission and to wait for coarse-grained timeout. On the other hand, TCP New Reno performs poorly by increasing the number of moving nodes, and it takes long end-to-end delay. Reason is limitation of retransmitting the single lost segment against per RTT, as a result a large delay occurs in retransmitting the later lost packets in the window. From other side, if the sender is restricted by the receiver's advertised window during recovery time, then the sender is unable to utilize the existing bandwidth successfully that takes long end-to-end delay [16]. Minimum end-to-end delay for TCP Vegas is fairness of retransmission algorithm when segment is lost that TCP Vegas waits for 2 dupack and retransmit the lost segments before expiry of timeouts. Furthermore, the RTT of Vegas shrinks only once during the RTT.

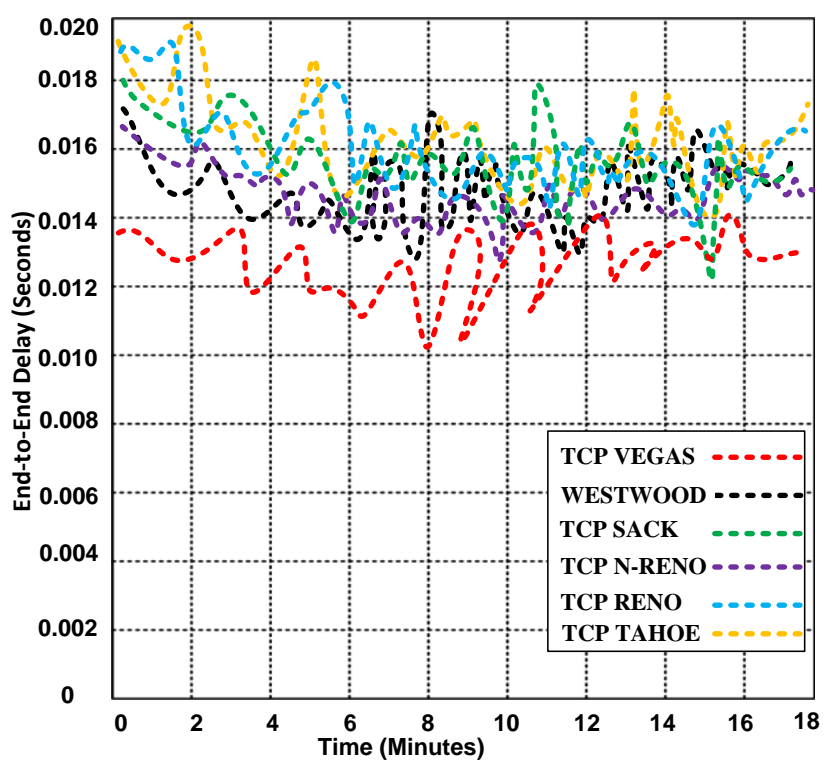

Fig 7: Average End-to-End delay of each TCP variants at the maximum speed of $20 \mathrm{~m} / \mathrm{sec}$ 


\subsection{Broken links at mobile node-speed}

We show an average broken links for each TCP variant at the speed of $10 \mathrm{~m} / \mathrm{sec}$ and $20 \mathrm{~m} / \mathrm{sec}$ given in Figure 8 . These broken links are calculated at the $50 \%$ moving nodes. When MANET nodes want to establish the sessions to obtain internet services from wired segment of the network then routing protocols start route discovery process. Route Request packet (RREQ) is broadcasted into network to obtain any single appropriate route to the destination.

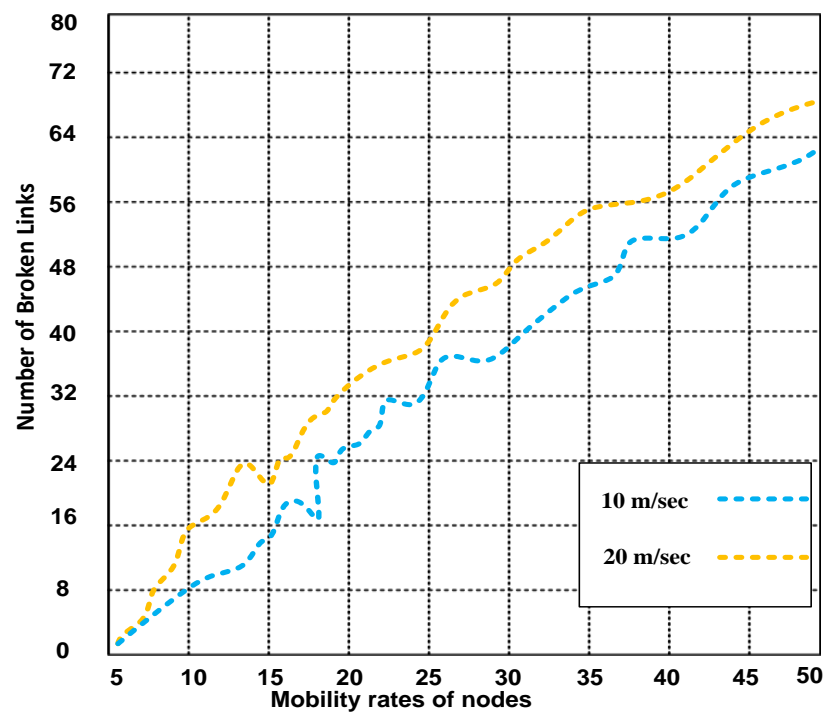

Fig 8: Broken links at $10 \mathrm{~m} / \mathrm{sec}$ and $20 \mathrm{~m} / \mathrm{sec}$

When route request packet is reached destination, in response route reply packet (RREP) is sent to originator RREQ. If a link is broken due to mobility and speed of intermediate nodes, a route error packet is sent to the destination. Meanwhile, destination finds another route. The process is repeated until the reply reaches to the targeted node. Therefore, destination finds another route if any error occurs in the current route.

This process causes delay in packet delivery. The high mobility and speed makes more broken links due to the discovery of the route. The high mobility and speed continuously change the direction of the node, in consequence more links break. Due to increase of speed, topology changes rapidly and more links are broken particularity in ODLW when more connections are established among the nodes.

\section{DISCUSSION OF RESULTS}

We have simulated TCP Variants in NS2 over heterogeneous network, which consists of MANET, wired \& wireless segments of the network. We have increased the mobility in MANET segment of the network by using ODLW routing protocol. Based on the simulation results, we analyzed that by increasing the mobility and speed of the nodes, the performance of each TCP variant gradually decreases. Multiple routes obviously give benefits, but creates disadvantage due to high mobility. In larger networks, the source routing principle can also generate a trouble. It has been observed that TCP Vegas performed better than other TCP variants. TCP Vegas produces healthy throughput, better in-order-delivery of data, minimum end- to-end delay, and good RTT at different mobility ratios and speeds. The major reasons for degraded performance of TCP Reno, Tahoe and New Reno are timeouts. As, during the timeouts period, they cannot resend the lost segments, whereas TCP Vegas does not wait for the loss to trigger [17].
Westwood greatly miscalculates the existing bandwidth, which is potentially troublesome for fairness. As a result, it can lead to starvation of simultaneous connections that is the reason to produce lesser throughput than TCP Vegas and also validated the reason in the work presented in [18]. Hence, Losses in wired network are due to overflow of the buffer at routers. TCP Reno, Tahoe and New Reno have been designed particularly for wired network and meet the requirement of IEEE802.11, but their performance become weaker in heterogeneous network especially satellite link is involved. Minimum mobility ratios create less control overhead, which causes the better performance for each TCP Variants, which is also proved in our simulation. TCP variants experience the most losses in multi-hop wireless networks, which are caused by packet drop at wireless link layer IEEE 802.11. To improve the performance, new congestion control flavors have been introduced, and various schemes are included.

Explicit congestion Notification (ECN) has been incorporated to improve the congestion control. If the congestion occurs in the network, then the intermediate routers will mark the congestion experience (CE) code point in the header of TCP [19]. The comparison of TCP variants is given in Table-2 that shows the strengths and weaknesses.

\section{CONCLUSION AND FUTURE WORK}

Mobile Adhoc networks can be deployed to different locations without the use of infrastructure support. In military environment, disaster situation, scattered educational institutions need such networks to route data packets through dynamically mobile nodes. MANET is a better choice for these extremely mobile and dynamic applications, which are not supported by centralized administration. If internet services are required that MANET is a better solution in anywhere to integrate with a wired network to construct as heterogeneous network to obtain an internet facility.

To investigate the performance of different transmission control protocols, we have conducted the simulation in NS-2 by using random waypoint mobility model and analyzed different metrics. We have particularly focused on MANET \& wired portions of the network to investigate the performance of TCP variants. The minimum effect of mobility has been analyzed on TCP Westwood and reasons are already discussed in detail but it delivers lesser segments than TCP Vegas and SACK whereas TCP Vegas has better throughput, minimum end-to-end delay, better in-order delivery of data and improved RTT. TCP SACK also performs better and does not loss many segments because sender is informed, which segment has been received. TCP SACK uses SACK blocks at receiver side to indicate the contiguous block of data successfully received.

The sender can find out through SACK blocks that segments are lost as this is the reason to control the loss of segments frequently. TCP Reno, TCP New Reno and Tahoe degrade the throughput in high mobility ratios and take more endto-end delay time as compared to other TCP variants. In future, we will analyze and evaluate TCP Variants in heterogeneous network with respect to different mobility models including social network model, random walk mobility model, random direction mobility model, city section mobility model, etc.

\section{REFERENCES}

[1] S. Zafar and B. B. F. Town, "A Survey of Transport Layer Protocols for Wireless Sensor Networks," 
International Journal of Computer Applications (09758887) Volume, vol. 33, 2011.

[2] Razaque, A., S. Banuri, K. Shahzad, M. A. Qadir, and M. Ahmad. "Analyis of TCP variants at various link error rates under mobility aware Anchor Point Node Hybrid Network." In Communications, Signals and Coding, 2008. MIC-CSC'08. Proceedings of the Mosharaka International Conference on, pp. 60-65. IEEE, 2008.

[3] Razaque, Abdul, Khurram Shahzad, and M. Abdul Qadir. "Performance Evaluation of TCP Variants in mobility based Anchor point node Hybrid Network." International IEEE/ACM proceedings on Communication Networks and Services Research (CNSR): 270-277.

[4] D. Kim, J.-C. Cano, P. Manzoni, and C. Toh, "A comparison of the performance of TCP-Reno and TCPVegas over MANETs," in Wireless Communication Systems, 2006. ISWCS'06. 3rd International Symposium on, 2006, pp. 495-499.

[5] F. Yunus, N.-S. Ismail, S. H. Ariffin, A. Shahidan, N. Fisal, and S. K. Syed-Yusof, "Proposed transport protocol for reliable data transfer in wireless sensor network (WSN)," in Modeling, Simulation and Applied Optimization (ICMSAO), 2011 4th International Conference on, 2011, pp. 1-7.

[6] O. Oyedapo and D. Ngwenya, "Evaluation of TCPvariants performances in an ad-hoc mobile network," in AFRICON 2007, 2007, pp. 1-7.

[7] S. Henna, "A throughput analysis of TCP variants in mobile wireless networks," in Next Generation Mobile Applications, Services and Technologies, 2009. NGMAST'09. Third International Conference on, 2009, pp. 279-284.

[8] A. Oluwatope, A. Obabire, G. Aderounm, and M. Adigun, "End-to-End Performance Evaluation of Selected TCP Variants across a Hybrid Wireless Network," Issues in Informing Science and Information Technology, vol. 3, 2006.

[9] S. Papanastasiou, M. Ould-Khaoua, and L. Mackenzie, "On the Evaluation of TCP in MANETs," in International Workshop on Wireless Ad Hoc Networking (IWWAN'05), 2005.

[10] M. Musolesi, S. Hailes, and C. Mascolo, "An ad hoc mobility model founded on social network theory," in Proceedings of the 7th ACM international symposium on Modeling, analysis and simulation of wireless and mobile systems, 2004, pp. 20-24.

[11] A. Lo, J. Zhou, M. Jacobsson, and I. Niemegeers, "ns-2 models for simulating a novel beyond $3 \mathrm{G}$ cellular multihop network," in Proceeding from the 2006 workshop on ns-2: the IP network simulator, 2006, p. 7.

[12] K. Yusung, C. Kilnam, and X. Lisong, "Adjusting the aggregate throughput of parallel TCP flows without central coordination," IEICE transactions on communications, vol. 91, pp. 1615-1618, 2008.

[13] D. Kim, H. Bae, J. Song, and J.-C. Cano, "Analysis of the interaction between TCP variants and routing protocols in MANETs," in Parallel Processing, 2005. ICPP 2005 Workshops. International Conference Workshops on, 2005, pp. 380-386.
[14] Z. Fu, H. Luo, P. Zerfos, S. Lu, L. Zhang, and M. Gerla, "The impact of multihop wireless channel on TCP performance," Mobile Computing, IEEE transactions on, vol. 4, pp. 209-221, 2005.

[15] S. Ha, Y. Kim, L. Le, I. Rhee, and L. Xu, "A step toward realistic performance evaluation of high-speed TCP variants," in Fourth International Workshop on Protocols for Fast Long-Distance Networks, 2006.

[16] H.-Y. Hsieh, K.-H. Kim, and R. Sivakumar, "An end-toend approach for transparent mobility across heterogeneous wireless networks," Mobile Networks and Applications, vol. 9, pp. 363-378, 2004.

[17] M. Hassan and R. Jain, High performance TCP/IP networking: Prentice Hall, 2003.

[18] S. Mascolo, L. A. Grieco, R. Ferorelli, P. Camarda, and G. Piscitelli, "Performance evaluation of Westwood+ TCP congestion control," Performance Evaluation, vol. 55, pp. 93-111, 2004.

[19] Razaque, A., K. Shahzad, and M. A. Qadir. "Analytical comparison of TCP Variants under mobility aware Anchor Point Node (APN) Hybrid Network." In Communications, Signals and Coding, 2008. MICCSC'08. Proceedings of the Mosharaka International Conference on, pp. 66-71. IEEE, 2008.

\section{BIOGRAPHY}

Abdul Razaque is Editor-in-Chief for International Journal for Engineering Technology (IJET) Singapore, He holds fellowship form Higher Education Commission (HEC) Pakistan, and Common Wealth, UK. He served as Head of computer science department in Model colleges setup Islamabad, Pakistan from 2002 to 2009. He also led several projects as project Director for promoting the trend of information technology (IT) in Pakistan funded by United Nation organization (UNO) and World Bank during 2005 to 2008. He is currently active researcher of wireless and Mobile communication (WMC) laboratory, UB, USA. Abdul Razaque has also been working as Chair, Strategic Planning Committee for IEEE SAC Region-1. USA and Relational Officer for IEEE SAC Region-1 for Europe, Africa and Middle-East. Abdul Razaque has chaired more than dozen of highly reputed international conferences and also delivered his lectures as Keynote Speaker. His research interests include the wireless sensor networks, design and development of learning environments, TCP/IP protocols, multimedia applications and ambient intelligence.

Dr. Khaled Elleithy is the Associate Vice President for Graduate Studies and Associate Dean for Graduate Studies in the School of Engineering at the University of Bridgeport. He has research interests are in the areas of network security, mobile communications, and formal approaches for design and verification. He has published around three hundred research papers in international journals and conferences in his areas of expertise. Dr. Elleithy is the co-chair of the International Joint Conferences on Computer, Information, and Systems Sciences, and Engineering (CISSE). CISSE is the first Engineering/Computing and Systems Research EConference in the world to be completely conducted online in real-time via the internet and was successfully running for six years. Dr. Elleithy is the editor or co-editor of 12 books published by Springer for advances on Innovations and Advanced Techniques in Systems, Computing Sciences and Software. 


\section{APPENDIX:}

Table 2: Benchmark of TCP Variants: Tahoe, Reno, New-Reno, SACK, Vegas, Westwoo

\begin{tabular}{|c|c|c|c|c|c|c|c|}
\hline $\begin{array}{c}\text { TCP } \\
\text { Variants }\end{array}$ & RTT & Throughput & $\begin{array}{c}\text { Average } \\
\text { Broken Links } \\
\text { with 50\% } \\
\text { mobile nodes }\end{array}$ & $\begin{array}{c}\text { End-to-End } \\
\text { Delay }\end{array}$ & $\begin{array}{c}\text { Mobility- } \\
\text { aware }\end{array}$ & Packet Drop \% & Efficiency \\
\hline Tahoe & 0.074 & $335 \mathrm{~KB} / \mathrm{Sec}$ & 34 & Moderate & Moderate & 0.0013 & 0.9987 \\
\hline Reno & 0.072 & $334.6 \mathrm{~KB} / \mathrm{Sec}$ & 35 & Moderate & Moderate & 0.0009 & 0.9991 \\
\hline New Reno & 0.069 & $336.1 \mathrm{~KB} / \mathrm{Sec}$ & 31 & Moderate & Moderate & 0.0008 & 0.9992 \\
\hline SACK & 0.070 & $332.9 \mathrm{~KB} / \mathrm{Sec}$ & 25 & Moderate & Moderate & 0.0011 & 0.9989 \\
\hline Vegas & 0.062 & $344.1 \mathrm{~KB} / \mathrm{Sec}$ & 14 & Low & High & 0.0006 & 0.9994 \\
\hline Westwood & 0.068 & $337.2 \mathrm{~KB} / \mathrm{Sec}$ & 19 & Low & High & 0.0007 & 0.9993 \\
\hline
\end{tabular}

